\title{
Article \\ Selection of Bacterial Strains for Control of Root-Knot Disease Caused by Meloidogyne incognita
}

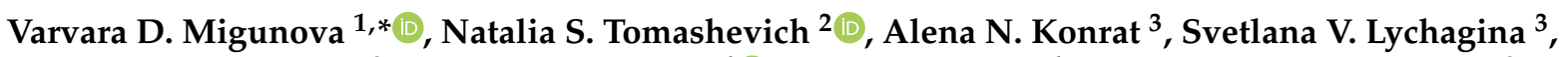 \\ Valentina M. Dubyaga ${ }^{2}$, Trifone $D^{\prime}$ Addabbo ${ }^{4} \mathbb{D}$, Nicola Sasanelli ${ }^{4}$ and Anzhela M. Asaturova ${ }^{2}$ \\ 1 A.N. Severtsov Institute of Ecology and Evolution, Russian Academy of Sciences, Leninsky Prospect 33, \\ 119071 Moscow, Russia \\ 2 Federal State Budgetary Scientific Institution, Federal Scientific Center of Biological Plant Protection (FSBSI \\ FSCBPP), 350039 Krasnodar, Russia; nataliatomashevich@yandex.ru (N.S.T.); dubyaga608@mail.ru (V.M.D.); \\ asaturovaanzhela@yandex.ru (A.M.A.) \\ 3 Federal State Budget Scientific Institution, Federal Scientific Centre VIEV (FSC VIEV) of RAS, Bolshaya \\ Cheryomushkinskaya 28, 117218 Moscow, Russia; alenakonrat@vniigis.ru (A.N.K.); \\ s.lychagina@vniigis.ru (S.V.L.) \\ 4 Institute for Sustainable Plant Protection, CNR, Via G. Amendola 122/D, 70126 Bari, Italy; \\ trifone.daddabbo@ipsp.cnr.it (T.D.); nicola.sasanelli@ipsp.cnr.it (N.S.) \\ * Correspondence: barbarusha@rambler.ru
}

\section{check for}

updates

Citation: Migunova, V.D.; Tomashevich, N.S.; Konrat, A.N.; Lychagina, S.V.; Dubyaga, V.M.; D'Addabbo, T.; Sasanelli, N.; Asaturova, A.M. Selection of Bacterial Strains for Control of Root-Knot Disease Caused by Meloidogyne incognita. Microorganisms 2021, 9, 1698. https://doi.org/10.3390/ microorganisms 9081698

Academic Editor: Ferenc Tóth

Received: 30 June 2021

Accepted: 6 August 2021

Published: 10 August 2021

Publisher's Note: MDPI stays neutral with regard to jurisdictional claims in published maps and institutional affiliations.

Copyright: (c) 2021 by the authors. Licensee MDPI, Basel, Switzerland. This article is an open access article distributed under the terms and conditions of the Creative Commons Attribution (CC BY) license (https:/ / creativecommons.org/licenses/by/ $4.0 /)$.

\begin{abstract}
Root-knot disease caused by Meloidogyne incognita leads to significant crop yield losses that may be aggravated by the association with pathogenic fungi and bacteria. Biological agents can be effectively used against the complex disease of root-knot nematode and pathogenic fungi. In this study, 35 bacterial strains were analyzed for their in vitro nematicidal, antagonistic and growth stimulation activities. Based on results from the in vitro assays, grow-box experiments on tomato and cucumber were carried out with the strain BZR 86 of Bacillus velezensis applied at different concentrations. Effects of B. velezensis BZR 86 on the development of root-knot disease were evaluated by recording root gall index, number of galls and number of eggs in egg masses. Application of B. velezensis BZR 86 noticeably decreased the development of root-knot disease on tomato and cucumber plants, as well as significantly increased growth and biomass of cucumber plants in accordance with bacterial concentration. This study seems to demonstrate that strain B. velezensis BZR 86 could be an additional tool for an environmentally safe control of root-knot disease on horticultural crops.
\end{abstract}

Keywords: Bacillus velezensis; Meloidogyne incognita; Fusarium; Rhizoctonia; root-knot nematodes; nematicidal activity; antagonistic activity; plant growth

\section{Introduction}

Root-knot nematodes (RKNs) are agents of severe root-knot disease (RKND) on many crops [1]. In Russia alone crop losses from RKND caused by the root-knot nematode Meloidogyne incognita Kofoid and White (Chitw.) ranged between 20\% and 80\% in cucumber and tomato plants cultivated under greenhouse conditions [2]. Symptoms of disease can be aggravated by the presence of bacterial and fungal pathogens using paths of RKNs penetration into plant roots [3-5], which means that control of this complex disease requires a suppression of RKNs and plant pathogenic microorganisms in soil, as well as stimulation of plant growth.

One of the environmentally safe methods for plant protection from RKND is application of microorganisms and/or products of their metabolism. Pesticides and growth regulators of microbial origin have proved their significant potential in sustainable agriculture and consequently in the development of green environment [6]. Microbial communities suppress RKNs, and genus Bacillus as a member of these communities functions as a biological agent, significantly decreasing the number of galls and egg masses of RKNs [7]. 
In particular, a significant role can be played by plant growth-promoting bacteria (PGPB), i.e., soil bacteria associated with plant roots reported for increasing plant resistance to biotic and environmental stresses and stimulating plant growth [8].

Application of PGPB has been repeatedly documented for reducing root-knot nematode infestations [9-13] and enhancing plant growth and yield at the same time [13-16]. RKND suppression by PGPB may involve different mechanisms such as competition, plant surface colonization, production of nematicidal and antimicrobial compounds (antibiotics, siderophores, hydrolytic enzymes, etc.), enhancement of host defense mechanisms [17-20].

Within PGPB, the most promising source of root-knot nematode control agents is represented by species of Bacillaceae family, widely occurring in soil and plant aerial parts and roots [21,22]. Most of available commercial products are formulations of Bacillus subtilis and B. amyloliquefaciens [23]. Moreover, Bacillus firmus I-1582 (Bf I-1582) and B. amyloliquefaciens FZB42 (now reclassified as a strain of B. velezensis [24]) have been approved for use against RKNs on vegetable crops in Europe [25]. Mechanisms of suppressiveness of these species to RKNs are related to egg colonization and degradation, as well as to the induction of a plant systemic resistance [26]. Moreover, a commercial formulation based on B. velezensis (Botrybel) is available as biofungicide [27].

The general aim of this study was to evaluate growth stimulation, nematicidal and antagonistic efficacy of bacterial isolates associated with rhizoshere and rhizoplane of plants and select the most promising strain for control of root knot disease. Its objectives were (i) to determine the effects of bacterial isolates on nematodes and phytopathogenic fungi: Fusarium oxysporum, F. graminearum and Rhizoctonia solani, (ii) to analyze the influence of bacterial isolates on development of plants, (iii) to find out if the selected strain can control the root knot disease.

\section{Materials and Methods}

\subsection{Bacterial Strains}

All the bacterial strains used in this study were isolated from soil, rhizoplane and rhizosphere of plants from Krasnodar region (Russian Federation) (Table S1). The isolation was done using the Warcup method and dilution technique [28]. The 35 strains tested in the in vitro assays were selected from the Bioresource Collection "State Collection of Entomoacariphages and Microorganisms" of Federal Scientific Center of Biological Plant Protection (Bioresource Collection of FSCBPP) according to their lipase, chitinase and protease activity (Table S2) $[29,30]$ as enzymatic activities may be involved in the control efficacy on plant parasitic nematodes [31]. In this research, we used the scientific equipment «Technological line for obtaining microbiological plant protection products of a new generation» (https: / / ckp-rf.ru/usu/671367/ (accessed on 1 July 2021)). Some of the isolates were identified by standard microbiological characterization [32] followed by $16 \mathrm{~S}$ rRNA gene sequence analysis [33], full genome sequence was obtained for the strains that had demonstrated high nematicidal and antagonistic activities, namely: BZR 86 (https:/ / www.ncbi.nlm.nih.gov/bioproject/PRJNA677970, (accessed on 12 November 2020)), BZR 277 (https: / /www.ncbi.nlm.nih.gov/bioproject/PRJNA677969, (accessed on 12 November 2020)) [34] and BZR 517 (https:/ / www.ncbi.nlm.nih.gov/assembly/GCA_ 009683155.1\#/def, (accessed on 12 November 2020)) [35]. The multiple alignment of concatenated amino acid sequences of 120 bacterial single-copy marker genes was carried out using the Genome Taxonomy Data Base (GTDB-Tk v.1.3.0 toolkit software) by RefSeq and Genbank genomes (U.S.A.) [36]. This multiple alignment was used to construct the maximum likelihood phylogenetic tree using PhyML v.3.3 [37], using default parameters. The level of support for internal branches was assessed using the Bayesian test in PhyML.

\subsection{Antagonistic Activity against Phytopathogenic Fungi}

Antagonistic activity of bacterial strains was determined by dual-culture plate method on potato glucose agar and King B medium [38]. A mycelial plug of Fusarium oxysporum, F. graminearum or Rhizoctonia solani was put in a Petri dish and a bacterial strain was plated at 
the distance of $6 \mathrm{~cm}$ from fungus. Control plates contained the fungus and bacterium alone. Cultures were incubated for 20 days at $28^{\circ} \mathrm{C}$. The growth of colonies was checked every day. The presence of sterile zone and its size, as well as fungal color, density and direction of mycelial growth were registered. Antagonistic activity was calculated according to the formula: $\%$ inhibition $=[1-($ Fungal growth $/$ Control growth $)] \times 100$ [39].

\subsection{In Vitro Nematicidal Activity}

The 35 bacterial strains were grown in liquid medium 925 [40], having the following composition: $3 \mathrm{~g} \mathrm{~L}^{-1} \mathrm{~K}_{2} \mathrm{HPO}_{4}, 1 \mathrm{~g} \mathrm{~L}^{-1}, \mathrm{NaH}_{2} \mathrm{PO}_{4}, 1 \mathrm{~g} \mathrm{~L}^{-1} \mathrm{NH}_{4} \mathrm{Cl}, 0.3 \mathrm{~g} \mathrm{~L}^{-1} \mathrm{MgSO}_{4}$, $10 \mathrm{~g} \mathrm{~L}^{-1}$ sucrose, $2 \mathrm{~g} \mathrm{~L}^{-1}$ peptone, $1 \mathrm{~L}$ water. Fungivorous nematode Paraphelenchus tritici was used as model organism for the preliminary screening of nematicidal activity. Nematode population was grown on the fungus Alternaria tenuis in Petri dishes and then extracted by the Baermann funnel technique [41,42]. A $0.5 \mathrm{~mL}$ of the P. tritici water suspension, containing 50 nematode specimens, was added to each well of 24-well plates and then added with a $0.5 \mathrm{~mL}$ of each bacteria suspension. The test was done in five replicates. Nematode mortality was detected microscopically after $24 \mathrm{~h}$.

Bacterial strains' activity on the second-stage juveniles (J2) of $M$. incognita was determined using bacterial suspensions grown in liquid medium 925 and their supernatants. Supernatants were obtained by centrifugation of bacterial suspension at 10,000 rpm for $10 \mathrm{~min}$ and did not contain any living bacterial cells. Bacterial suspension at concentration $10^{8} \mathrm{CFU} \mathrm{mL}{ }^{-1}$ was diluted 10, 50, 100, 1000 and 10,000 times. A $0.5 \mathrm{~mL}$ amount of the obtained preparation of tested bacteria or supernatants was pipetted into 24-well plate with $0.5 \mathrm{~mL}$ of viable $J 2$ of $M$. incognita (100 ind.). Nematicidal effect was monitored after 24 and $48 \mathrm{~h}$, after which nematodes were placed in sterile water for further $24 \mathrm{~h}$ to check for possible nematostatic effect. $J 2$ mortality was calculated according to the the Schneider Orelli's formula: Corrected \% Mortality $=([$ mortality \% in treatment - mortality $\%$ in control] $/[100-$ mortality $\%$ in control] $) \times 100$ [43]. The test was done twice in eight replicates.

\subsection{In Vitro Activity on Plant Growth}

Thirty wheat seeds (cv. Raduga) were placed in Petri dishes with gauze, treated with $15 \mathrm{~mL}$ of bacterial suspensions and grown for $72 \mathrm{~h}$ at $25^{\circ} \mathrm{C}$. Effects of bacterial strains on plant growth was evaluated by the germination index (GI), i.e., the ratio between length of treated wheat seedlings and non-treated seedlings.

\subsection{Pot Experiments}

\subsubsection{Effects on Wheat Plants}

Seeds of winter wheat (cv. Batko) not sterilized were soaked for $2 \mathrm{~h}$ in two-day bacterial cultures $10^{9} \mathrm{CFU} \mathrm{mL} \mathrm{mL}^{-1}$ obtained by washing bacteria from Petri dishes followed by adding tap water to reach the volume of $50 \mathrm{~mL}$. After $2 \mathrm{~h}$, seeds were removed from bacterial suspensions and dried on filter paper. After $20-24 \mathrm{~h}, 30$ seeds were sown in each of the three $0.45 \mathrm{~L}$ pots filled with sterilized sand. In total 90 seeds were planted, of which 80 germinated. The pots were stored in greenhouse at $24-28^{\circ} \mathrm{C}$ and 11,000 Lux. The length of roots, height of stems and plant biomass were measured after 14 days. The testing of root length and stem height was done in 80 replicates. As for the biomass of the plants, we measured it by weighing each of the three pots because of the small size of individual plants. The experiment was performed twice.

\subsubsection{Effects on RKND and Plant Growth in Grow-Box Experiment}

Strain BZR 86 was selected for the experiments in soil infested by RKN. A 1:1 mixture of peat and sand was poured in $0.18 \mathrm{~L}$ plastic pots, which were then sown with three-weekold cucumber seedlings cv. Kurazh (parthenocarpic) with three true leaves; the second test was done with 68-day-old tomato seedlings cv. Balkonnoe chudo. Two separated pot experiments with cucumber and tomato plants were conducted under grow-box conditions 
in All-Russian Scientific Research Institute for Fundamental and Applied Parasitology of Animals and Plants (Moscow, Russia). A M. incognita population, originally collected in Krasnodar region (Russian Federation) was reared on tomato (cv. Balkonnoe chudo) roots for 70 days, after which nematode egg-masses were picked and incubated in sterile water at $25{ }^{\circ} \mathrm{C}$. The emerged $J 2$ were pipetted into each pot at a density of $150 \mathrm{~J} 2 /$ pot. A bacterial culture of $B$. velezensis BZR 86 , resulted as the most effective strain in the in vitro screenings, was prepared by fermentation in flasks in the medium 925 . The culture was maintained for $45 \mathrm{~h}$ at $29^{\circ} \mathrm{C}$ under shaking $(190 \mathrm{rpm})$, as to reach about $10^{8}-10^{9} \mathrm{CFU} \mathrm{mL}{ }^{-1}$ concentration. A $50 \mathrm{~mL}$ volume of bacterial suspension diluted in tap water was added to each pot, as to reach three different test concentrations: maximal: $(3-7) \times 10^{6} \mathrm{CFU} \mathrm{mL}^{-1}$ of soil substrate, medium: (3-7) $\times 10^{5} \mathrm{CFU} \mathrm{mL}{ }^{-1}$ of soil substrate and minimal: $(3-7) \times 10^{4} \mathrm{CFU} \mathrm{mL}^{-1}$ of soil substrate. Concentrations of bacterial suspensions were determined by counting the colony forming units (CFU) on Luria-Bertrani agar (Sigma). Non-treated soil, either infested and non-infested with $M$. incognita, and infested soil treated with the chemical standard Phytoverm (avermectin C, $2 \mathrm{gL}^{-1}$ ) were used as controls. Seven replicates were provided for each treatment.

Pots were maintained at $25^{\circ} \mathrm{C}$ under grow-box conditions for two months. At the end of the experiments, the height, number of leaves, ovaries, weight of aerial part and root biomass and root volume (as water displacement) were measured on each plant. Effects of $B$. velezensis BZR 86 on RKN infestation were determined by estimating the root gall index (RGI) according to a $0-5$ scale, in which: $0=$ no galls; $1=0.1 \%-10 \% ; 2=11 \%-35 \%$; $3=36 \%-70 \% ; 4=$ more than $70 \% ; 5=$ dead plant [41], as well as by microscopically counting number of eggs in egg masses.

\subsection{Statistical Analysis}

Data from the experiments were subjected to analysis of variance (ANOVA) and means compared by Duncan's multiple range test $(p<0.05)$. All statistical analyses were performed using Microsoft Excel (standard deviation) and Statistica Version 13.5.0.17.T. (ANOVA, normality of data and Duncan's multiple range test).

\section{Results}

\subsection{Characterization of Bacterial Strains}

Nematicidal activity varied from $0 \%$ to $100 \%$ among the 35 bacterial strains, though $46 \%$ showed a nematicidal activity higher than $85 \%$ (Table 1). The antagonistic activity against phytopathogenic fungi varied from 0 to $58 \%$ though only 14 of the studied strains showed a simultaneous antagonistic activity against Fusarium graminearum, F. oxysporum and Rhizoctonia solani higher than $30 \%$. GI values ranged from 0.22 to 1.23 , but only $28 \%$ of strains demonstrated a growth stimulating effect $(\mathrm{GI}>1)$.

Only four strains simultaneously presented a high nematicidal effect $(100 \%)$, an antagonistic activity against $F$. graminearum, F. oxysporum and $R$. solani $(>30 \%)$ and a growth stimulation effect $(\mathrm{GI}>1)$. These four strains were Bacillus species identified as BZR 86 , BZR 623, BZR 261 and BZR 441.

In the pot experiment on wheat, significant effects on plant root length and height occurred for strains BZR 441, BZR 623 and BZR 261 (Table S3, Table 2). These effects were both negative (strain BZR 441) and positive (strains BZR 623 and BZR 261). Conversely, no significant difference from the control was found for BZR 86. 
Table 1. Germination index, nematicidal and antagonistic effects of bacterial strains selected from the Bioresource Collection of FSCBPP.

\begin{tabular}{|c|c|c|c|c|c|}
\hline \multirow{2}{*}{ Strain ${ }^{1}$} & \multirow{2}{*}{$\begin{array}{l}\text { Nematicidal } \\
\text { Activity (\%) }\end{array}$} & \multirow{2}{*}{$\mathrm{GI}^{2}$} & \multicolumn{3}{|c|}{ Antagonistic Activity on the 15th Day (\%) } \\
\hline & & & F. oxysporum & R. solani & F. graminearum \\
\hline BZR 18 & $92 \pm 3.1^{3} \mathrm{ij}^{4}$ & $0.84 \pm 0.06$ efg & $0 \pm 0 \mathrm{k}$ & $0 \pm 0 \mathrm{k}$ & $53.9 \pm 2.2 \mathrm{bcd}$ \\
\hline BZR 59 & $63 \pm 0$ hi & $0.86 \pm 0.23 \mathrm{efgh}$ & $0 \pm 0 \mathrm{k}$ & $28 \pm 1.3 \mathrm{ij}$ & $52.9 \pm 1.4 \mathrm{~cd}$ \\
\hline BZR 86 & $100 \pm 5 \mathrm{j}$ & $1.18 \pm 0.06 \mathrm{mn}$ & $32.4 \pm 2 \mathrm{f}$ & $45.8 \pm 0.8 \mathrm{bcd}$ & $45.9 \pm 0.8 \mathrm{~h}$ \\
\hline BZR 148 & $4 \pm 0$ ef & $0.78 \pm 0.08$ def & $42.2 \pm 0.8 \mathrm{a}$ & $48.4 \pm 0.8 \mathrm{a}$ & $41.5 \pm 1.4 \mathrm{ij}$ \\
\hline BZR 187 & $100 \pm 0 \mathrm{j}$ & $0.94 \pm 0.08$ ghij & $40 \pm 1.3 \mathrm{c}$ & $43.1 \pm 1.5 \mathrm{e}$ & $32.2 \pm 0.8 \mathrm{mn}$ \\
\hline BZR 241 & $96 \pm 0 \mathrm{j}$ & $0.87 \pm 0.08$ efghi & $41.8 \pm 0.8 \mathrm{abd}$ & $43.1 \pm 3.4 \mathrm{e}$ & $41 \pm 0.8 \mathrm{ij}$ \\
\hline BZR245 F & $12 \pm 0.6 \mathrm{bcd}$ & $0.44 \pm 0.09 \mathrm{~b}$ & $25.8 \pm 2.0 \mathrm{i}$ & $25.8 \pm 2.0 \mathrm{j}$ & $28 \pm 0.8 \mathrm{o}$ \\
\hline BZR 261 & $100 \pm 0.5 \mathrm{j}$ & $1.10 \pm 0.09 \mathrm{klmn}$ & $36.4 \pm 0.8 \mathrm{e}$ & $46.7 \mathrm{ab}$ & $39.5 \pm 0.8 \mathrm{j}$ \\
\hline BZR 277 & $92 \pm 0 \mathrm{ij}$ & $0.22 \pm 0 \mathrm{a}$ & $40.9 \pm 0.8 \mathrm{abcd}$ & $44 \pm 2.7$ cde & $42.9 \pm 1.4 \mathrm{i}$ \\
\hline BZR 337 & $70 \pm 0 \mathrm{~g}$ & $0.67 \pm 0.05 \mathrm{~cd}$ & $0 \pm 0 \mathrm{k}$ & $0 \pm 0 \mathrm{k}$ & $52.5 \pm 0.8 \mathrm{~cd}$ \\
\hline BZR 348 & $17 \pm 0 \mathrm{~cd}$ & $0.73 \pm 0.10$ cde & $24 \pm 2.3 \mathrm{j}$ & $46.7 \mathrm{ab}$ & $35.9 \pm 2.5 \mathrm{kl}$ \\
\hline BZR 367 & $0 \pm 0 \mathrm{a}$ & $1.00 \pm 0$ hijkl & $25.3 \pm 2.3 \mathrm{ij}$ & $28.4 \pm 2.0 \mathrm{i}$ & $30.8 \pm 2.2 \mathrm{n}$ \\
\hline BZR 413 & $82 \pm 0 \mathrm{ij}$ & $1.20 \pm 0.07 \mathrm{n}$ & $0 \pm 0 \mathrm{k}$ & $0 \pm 0 \mathrm{k}$ & $31.8 \pm 0.8 \mathrm{n}$ \\
\hline BZR 416 & $16 \pm 1.7 \mathrm{~cd}$ & $0.93 \pm 0.10$ ghij & $25.8 \pm 0.8 \mathrm{i}$ & $25.8 \pm 0.8 \mathrm{j}$ & $28.3 \pm 0.8 \mathrm{o}$ \\
\hline BZR 417 & $12 \pm 0$ de & $1.02 \pm 0.05 \mathrm{ijkl}$ & $39.6 \pm 0.8 c$ & $40 \pm 3.5 \mathrm{f}$ & $39.9 \pm 1.4 \mathrm{j}$ \\
\hline BZR 430 & $10 \pm 0 \mathrm{bc}$ & $1.02 \pm 0.09 \mathrm{ijkl}$ & $0 \pm 0 \mathrm{k}$ & $0 \pm 0 \mathrm{k}$ & $42.4 \pm 0.8 \mathrm{i}$ \\
\hline BZR 436 & $7 \pm 0 \mathrm{~cd}$ & $0.84 \pm 0.29$ efg & $28 \pm 1.3 \mathrm{gh}$ & $32 \pm 2.3 \mathrm{~h}$ & $50 \pm 1.4$ ef \\
\hline BZR 441 & $100 \pm 0 j$ & $1.06 \pm 0.08 \mathrm{jklm}$ & $39.1 \pm 2.0 \mathrm{c}$ & $43.6 \pm 0.8 \mathrm{de}$ & $54 \pm 1.4 b c$ \\
\hline BZR 455 & $100 \pm 0 \mathrm{j}$ & $1.13 \pm 0.07 \mathrm{lmn}$ & $0 \pm 0 \mathrm{k}$ & $0 \pm 0 \mathrm{k}$ & $58.1 \pm 2.2 \mathrm{a}$ \\
\hline BZR 462 & $93 \pm 11.5 \mathrm{ij}$ & $1.00 \pm 0$ hijkl & $42.2 \pm 0.8 \mathrm{ab}$ & $47.1 \pm 0.8 \mathrm{ab}$ & $36.9 \pm 0.8 \mathrm{k}$ \\
\hline BZR 472 & $92 \pm 3.5 \mathrm{ij}$ & $0.68 \pm 0.07 \mathrm{~cd}$ & $0 \pm 0 \mathrm{k}$ & $0 \pm 0 \mathrm{k}$ & $48.5 \pm 1.4 \mathrm{fg}$ \\
\hline BZR 480 & $100 \pm 0 j$ & $1.00 \pm 0$ hijkl & $0 \pm 0 \mathrm{k}$ & $0 \pm 0 \mathrm{k}$ & $34.3 \pm 1.4 \mathrm{~lm}$ \\
\hline BZR 512 & $100 \pm 0 \mathrm{j}$ & $0.63 \pm 0 c$ & $0 \pm 0 \mathrm{k}$ & $0 \pm 0 \mathrm{k}$ & $48 \pm 0.8 \mathrm{fg}$ \\
\hline BZR 517 & $80 \pm 0 \mathrm{hi}$ & $0.67 \pm 0.17 \mathrm{~cd}$ & $36.9 \pm 0.8 \mathrm{e}$ & $43.6 \pm 0.8 \mathrm{de}$ & $52.5 \pm 0.8 \mathrm{~cd}$ \\
\hline BZR 519 & $100 \pm 0.5 j$ & $0.94 \pm 0.08$ ghij & $40 \pm 0 \mathrm{~cd}$ & $46.2 \pm 4.1 \mathrm{abc}$ & $54.9 \pm 1.6 \mathrm{bc}$ \\
\hline BZR 523-1 & $84 \pm 4.2 \mathrm{hi}$ & $0.89 \pm 0$ fghi & $26.7 \pm 0 \mathrm{hi}$ & $43.6 \pm 0.8 \mathrm{de}$ & $51.5 \mathrm{de}$ \\
\hline BZR 523-2 & $16 \pm 0 \mathrm{~cd}$ & $0.89 \pm 0.11$ fghi & $0 \pm 0 \mathrm{k}$ & $0 \pm 0 \mathrm{k}$ & $48.5 \pm 1.4 \mathrm{fg}$ \\
\hline BZR 528 & $100 \pm 0 \mathrm{j}$ & $0.89 \pm 0.06$ fghi & $0 \pm 0 \mathrm{k}$ & $0 \pm 0 \mathrm{k}$ & $52.5 \pm 1.4 \mathrm{~cd}$ \\
\hline BZR 538 & $11 \pm 2.5 \mathrm{bc}$ & $0.75 \pm 0$ cdef & $0 \pm 0 \mathrm{k}$ & $0 \pm 0 \mathrm{k}$ & $45.3 \pm 1.4 \mathrm{~h}$ \\
\hline BZR 623 & $100 \pm 0.6 j$ & $1.10 \pm 0.09 \mathrm{klmn}$ & $40.4 \pm 0.8 \mathrm{bcd}$ & $48.4 \pm 0.8 \mathrm{a}$ & $41 \pm 0.8 \mathrm{ij}$ \\
\hline BZR 658 & $100 \pm 0 \mathrm{j}$ & $1.23 \pm 0.09 \mathrm{n}$ & $0 \pm 0 \mathrm{k}$ & $0 \pm 0 \mathrm{k}$ & $39.5 \pm 0.8 \mathrm{j}$ \\
\hline BZR 673 & $9 \pm 0$ de & $0.96 \pm 0.06$ ghijk & $42.2 \pm 2.0 \mathrm{ab}$ & $47.1 \pm 0.8 \mathrm{ab}$ & $47.1 \pm 1.4 \mathrm{gh}$ \\
\hline BZR 854 & $36 \pm 0 \mathrm{f}$ & $0.86 \pm 0.21$ efgh & $39.1 \pm 0.8 c$ & $48 \pm 1.3 \mathrm{ab}$ & $55.4 \pm 0.8 \mathrm{~b}$ \\
\hline BZR 862 & $96 \pm 0 j$ & $0.78 \pm 0$ def & $28.4 \pm 0.8 \mathrm{~g}$ & $34.7 \pm 1.3 \mathrm{~g}$ & $41.9 \pm 0.8 \mathrm{ij}$ \\
\hline BZR 873 & $77 \pm 19.6 \mathrm{gh}$ & $1.23 \pm 0.09 n$ & $0 \pm 0 \mathrm{k}$ & $0 \pm 0 \mathrm{k}$ & $52.9 \pm 1.4 \mathrm{~cd}$ \\
\hline
\end{tabular}

${ }^{1}$ Accession number of bacteria in the Bioresource Collection of FSCBPP; ${ }^{2}$ GI-germination index; ${ }^{3}$ average \pm SD of eight replicates; ${ }^{4}$ data flanked in each column by the same letters are not statistically different according to Duncan's multiple range test $(p=0.05)$.

Table 2. Effect of bacterial strains on growth and biomass of winter wheat plants (cv. Batko) in the pot experiment.

\begin{tabular}{ccccc}
\hline & $\begin{array}{c}\text { Plant Height } \\
\text { Trm) }\end{array}$ & $\begin{array}{c}\text { Root Length } \\
\mathbf{( c m )}\end{array}$ & \multicolumn{2}{c}{$\begin{array}{c}\text { Weight of Dry Biomass } \\
\text { (g) }\end{array}$} \\
\cline { 3 - 5 } & & & Aerial Parts & Roots \\
\hline Control & $14.6 \pm 1.8^{1} \mathrm{a}^{2}$ & $18.3 \pm 3.7 \mathrm{a}$ & $0.11 \pm 0 \mathrm{a}$ & $0.15 \pm 0.01 \mathrm{~d}$ \\
BZR 441 & $14.8 \pm 1.7 \mathrm{a}$ & $16.4 \pm 2.9 \mathrm{~b}$ & $0.11 \pm 0.01 \mathrm{a}$ & $0.13 \pm 0.01 \mathrm{bcd}$ \\
Control & $14.9 \pm 1.6 \mathrm{a}$ & $14.6 \pm 2.1 \mathrm{c}$ & $0.11 \pm 0.01 \mathrm{a}$ & $0.12 \pm 0.02 \mathrm{ab}$ \\
BZR 623 & $15.8 \pm 1.9 \mathrm{~b}$ & $14.5 \pm 3.1 \mathrm{c}$ & $0.12 \pm 0.01 \mathrm{a}$ & $0.14 \pm 0.01 \mathrm{~cd}$ \\
Control & $14.8 \pm 1.9 \mathrm{a}$ & $14.6 \pm 3.2 \mathrm{c}$ & $0.11 \pm 0.01 \mathrm{a}$ & $0.10 \pm 0.01 \mathrm{a}$ \\
BZR 86 & $15.1 \pm 1.7 \mathrm{a}$ & $14.5 \pm 3.0 \mathrm{c}$ & $0.11 \pm 0.01 \mathrm{a}$ & $0.13 \pm 0.01 \mathrm{bcd}$ \\
Control & $14.8 \pm 1.9 \mathrm{a}$ & $14.6 \pm 3.2 \mathrm{c}$ & $0.11 \pm 0 \mathrm{a}$ & $0.10 \pm 0.01 \mathrm{a}$ \\
BZR 261 & $14.7 \pm 1.9 \mathrm{a}$ & $17.0 \pm 3.6 \mathrm{~b}$ & $0.11 \pm 0 \mathrm{a}$ & $0.13 \pm 0.01 \mathrm{bc}$ \\
\hline
\end{tabular}

\footnotetext{
${ }^{1}$ Each value is an average \pm SD of six replicates (30 plants/replicate) from two independent experiments; ${ }^{2}$ data followed by the same letters are not statistically different according to Duncan's multiple range test $(p=0.05)$.
} 
Phylogenetic tree based on the whole genome sequencing of 120 conserved marker genes shows that strain BZR 86, selected according to results from the in vitro screenings, distinctly clusters with B. velezensis strain NRRL B-41580 (B. velezensis GCF 001461825.1 on the phylogenetic tree); the average nucleotide identity (ANI) value is $97.59 \%$ (Figure 1). The strain BZR 86 is related more closely to B. siamensis and B. amyloliquefaciens than to $B$. subtilis (as was determined by $16 \mathrm{~S}$ rRNA).

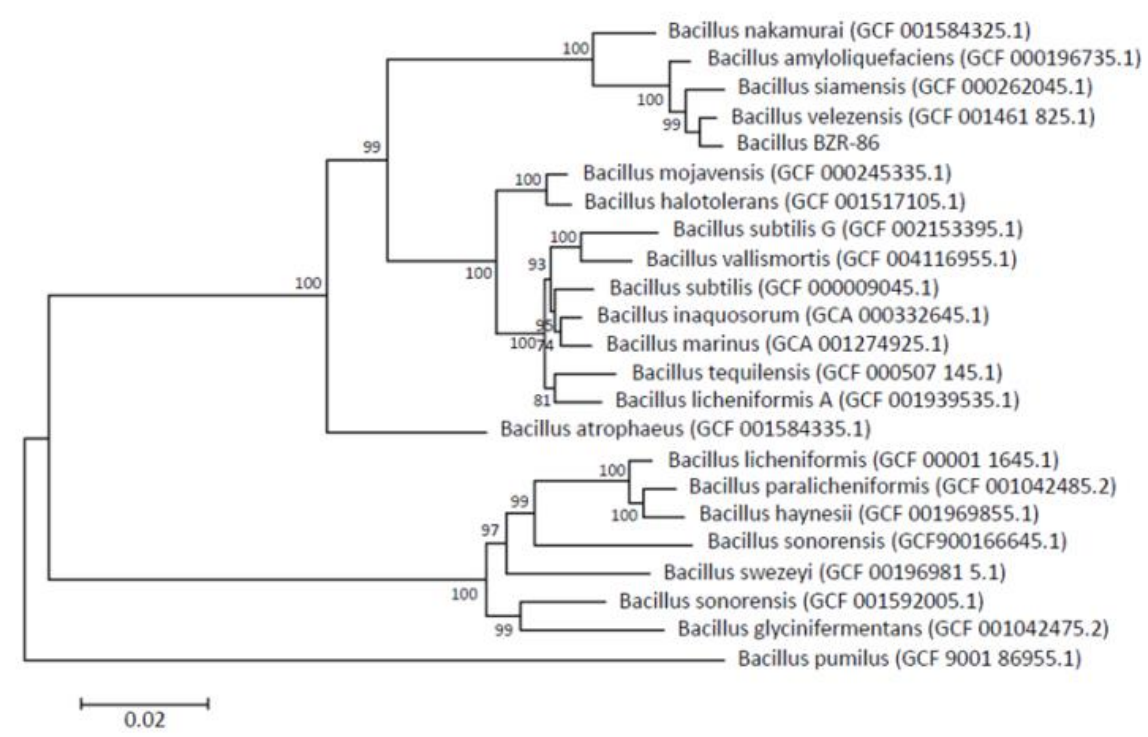

Figure 1. Maximum likelihood phylogenetic tree constructed using amino acid sequences of 120 conserved marker genes. The tree was constructed using PhyML v.3.3.

Figure 2 presents nematicidal activity of $B$. velezensis BZR 86 against $J 2$ of $M$. incognita. $\mathrm{LD}_{50}$ and $\mathrm{LD}_{99}$ of $J 2$ were $0.8 \times 10^{6}$ and $6.8 \times 10^{6} \mathrm{CFUmL}^{-1}$, respectively.

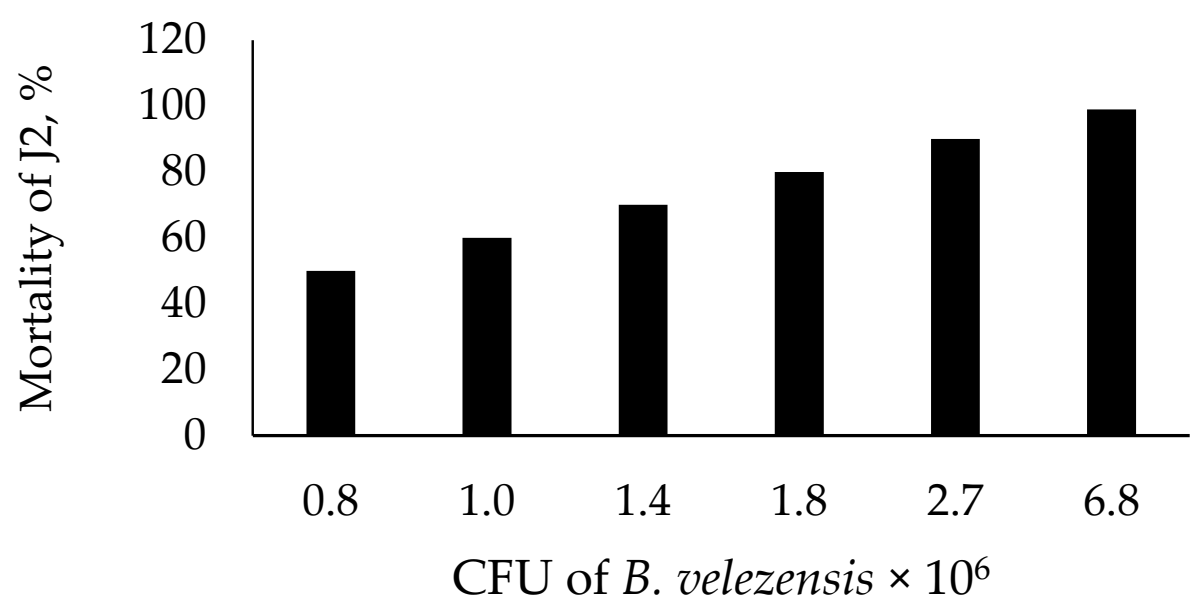

Figure 2. Nematicidal effect of Bacillus velezensis BZR 86 on J2 of Meloidogyna incognita.

Its antagonistic activity against F. graminearum, F. oxysporum and R. solani was $45.9 \%$, $32.4 \%$ and $45.8 \%$, respectively (Table 1, Figure 3 ). 


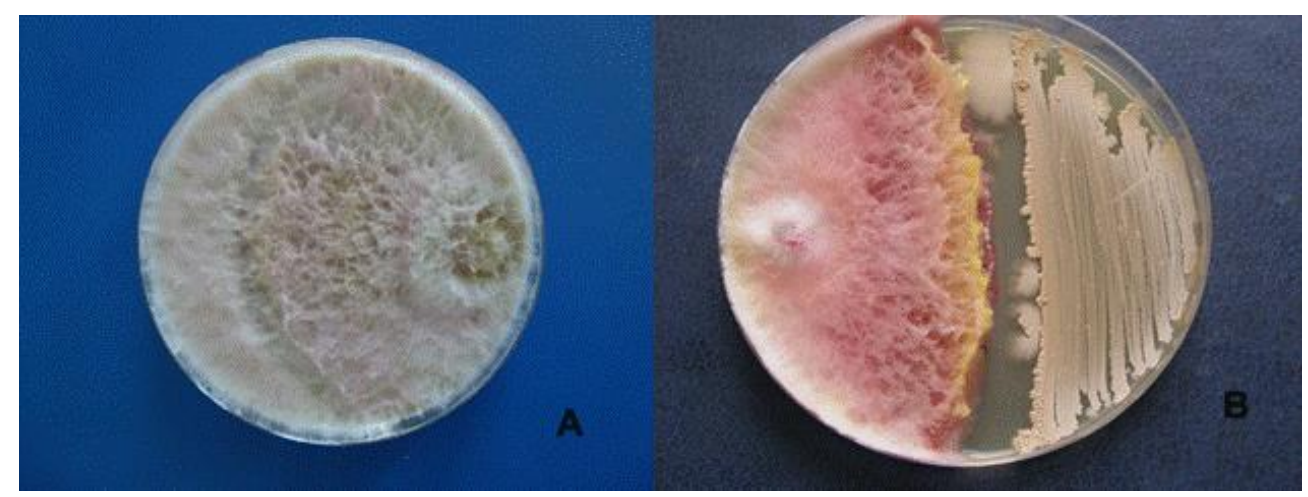

Figure 3. Antagonistic effect of Bacillus velezensis BZR 86 on Fusarium graminearum. (A)—control, (B)-Bacillus velezensis BZR 86.

\subsection{Influence of B. velezensis BZR 86 on development of the Root Knot Disease}

In the non-treated control, an attack of $M$. incognita led to a moderate damage both on cucumber and tomato plants, as root gall index varied from 2.6 to 2.8 (Tables 3 and 4). The experiments showed that $B$. velezensis BZR 86 influenced the development of root-knot disease on cucumber and tomato plants, reducing root gall index, number of galls and eggs in egg masses when applied to soil substrate without significant differences from chemical control Phytoverm (Tables 3 and 4).

Table 3. Influence of Bacillus velezensis BZR 86 on the development of root-knot disease of cucumber (cv. Kurazh).

\begin{tabular}{cccc}
\hline Treat & Galls/Root & Eggs/Egg Mass & $\begin{array}{c}\text { Root Gall Index } \\
\text { (1-5) }\end{array}$ \\
\hline Control & $218 \pm 58.6^{1} \mathrm{c}^{2}$ & $316 \pm 41.9 \mathrm{a}$ & $2.6 \pm 1.1 \mathrm{~b}$ \\
\hline Phytoverm & $73 \pm 13.2 \mathrm{ab}$ & $191 \pm 19.0 \mathrm{~b}$ & $1 \pm 0.6 \mathrm{a}$ \\
\hline BZR 86, 7 $\times 10^{6} \mathrm{CFU} \mathrm{mL}^{-1}$ & $86 \pm 16.8 \mathrm{ab}$ & $207 \pm 36.1 \mathrm{~b}$ & $1.4 \pm 0.9 \mathrm{a}$ \\
\hline BZR 86, 7 $\times 10^{5} \mathrm{CFU} \mathrm{mL}^{-1}$ & $141 \pm 31.8 \mathrm{bc}$ & $177 \pm 24.4 \mathrm{~b}$ & $1.8 \pm 0.5 \mathrm{ab}$ \\
\hline BZR 86, 7 $\times 10^{4} \mathrm{CFU} \mathrm{mL}^{-1}$ & $65 \pm 16.0 \mathrm{ab}$ & $224 \pm 33.9 \mathrm{~b}$ & $1.64 \pm 0.7 \mathrm{a}$ \\
\hline
\end{tabular}

${ }^{1}$ Each value is an average \pm SD of seven replicates. ${ }^{2}$ Data flanked in each column by the same letters are not statistically different according to Duncan's multiple range test $(p=0.05)$.

In the experiment on cucumber, the number of galls and the root gall index were significantly lower than at $7 \times 10^{4}$ and $7 \times 10^{6} \mathrm{CFU} \mathrm{mL} \mathrm{m}^{-1}$ of substrate concentrations of B. velezensis BZR 86 . All three concentrations of $B$. velezensis BZR 86 significantly reduced the number of eggs in egg masses (Table 3). The application of bacteria to soil substrate influenced the growth and development of cucumber plants. At the end of the experiment, considerable difference (almost double) in height was detected between the application of B. velezensis BZR 86 at $7 \times 10^{6} \mathrm{CFU} \mathrm{mL} \mathrm{mL}^{-1}$ of substrate concentration and control, Phytoverm and non-infested soil. Moreover, at the highest concentration of $B$. velezensis BZR 86, the number of plant leaves was significantly greater compared to Phytoverm and non-infested control.

By application of bacterium at the highest concentration, the biomass of the aerial part of cucumber plants increased twice compared with control, chemical standard and that at the lowest concentration (Table 5). 
Table 4. The influence of Bacillus velezensis BZR86 on the growth and infestation of Meloidogyne incognita on tomato (cv. Balkonnoe chudo).

\begin{tabular}{|c|c|c|c|c|c|c|c|}
\hline \multirow[t]{2}{*}{ Treatment } & \multirow{2}{*}{$\begin{array}{l}\text { Height } \\
\text { (cm) }\end{array}$} & \multirow[t]{2}{*}{$\mathbf{N}^{\circ}$ Leaves } & \multicolumn{2}{|c|}{$\begin{array}{c}\text { Plant Biomass } \\
\text { (g) }\end{array}$} & \multirow{2}{*}{$\begin{array}{l}\text { Root Volume } \\
\quad(\mathrm{mL})\end{array}$} & \multirow{2}{*}{$\begin{array}{c}\text { Root Gall } \\
\text { Index } \\
(0-5)\end{array}$} & \multirow[t]{2}{*}{ Galls/Root } \\
\hline & & & Aerial Part & Roots & & & \\
\hline $\begin{array}{c}\text { Non-infested } \\
\text { control }\end{array}$ & $16.2 \pm 2.4^{1} \mathrm{a}^{2}$ & $12 \pm 1.0 \mathrm{ab}$ & $5.0 \pm 1.6 \mathrm{a}$ & $1.0 \pm 0 \mathrm{~d}$ & $1.2 \pm 0.4 \mathrm{c}$ & - & - \\
\hline Infested control & $25.3 \pm 2.3 b$ & $15 \pm 1.9 \mathrm{~b}$ & $8.8 \pm 2.2 \mathrm{ab}$ & $3.5 \pm 1.0 \mathrm{ab}$ & $4.0 \pm 0.8 \mathrm{~b}$ & $2.8 \pm 1.0 \mathrm{~b}$ & $81 \pm 10.1 b$ \\
\hline Phytoverm & $20.0 \pm 3.4 \mathrm{ab}$ & $12 \pm 0.9 \mathrm{a}$ & $9.2 \pm 1.5 b$ & $2.2 \pm 0.8 c$ & $2.8 \pm 1.0 \mathrm{a}$ & $0.1 \pm 0 \mathrm{a}$ & $3 \pm 1.5 \mathrm{a}$ \\
\hline $\begin{array}{c}\text { BZR } 86,3 \times 10^{6} \\
\text { CFU mL }^{-1}\end{array}$ & $24.0 \pm 3.9 \mathrm{~b}$ & $12 \pm 2.0 \mathrm{ab}$ & $10.8 \pm 1.0 \mathrm{~b}$ & $3.8 \pm 1.0 \mathrm{~b}$ & $3.5 \pm 0.6 \mathrm{ab}$ & $0.1 \pm 0.1 \mathrm{a}$ & $2 \pm 1.7 \mathrm{a}$ \\
\hline $\begin{array}{c}\text { BZR } 86,3 \times 10^{5} \\
\text { CFU mL } \mathrm{mL}^{-1}\end{array}$ & $23.2 \pm 1.2 b$ & $15 \pm 3.7 \mathrm{ab}$ & $9.7 \pm 2.0 \mathrm{~b}$ & $3.5 \pm 0.5 \mathrm{ab}$ & $4.0 \pm 0.6 \mathrm{~b}$ & $0.1 \pm 0.1 \mathrm{a}$ & $1.2 \pm 0.8 \mathrm{a}$ \\
\hline $\begin{array}{l}\text { BZR 86, } 3 \times 10^{4} \\
\quad \text { CFU mL }^{-1}\end{array}$ & $21.3 \pm 5.3 \mathrm{ab}$ & $15 \pm 2.9 \mathrm{ab}$ & $8.1 \pm 1.2 \mathrm{ab}$ & $2.4 \pm 1.0 \mathrm{ac}$ & $2.9 \pm 1.1 \mathrm{a}$ & $0.3 \pm 0.4 \mathrm{a}$ & $8 \pm 2.7 \mathrm{a}$ \\
\hline
\end{tabular}

${ }^{1}$ Each value is an average \pm SD of seven replicates. ${ }^{2}$ Data flanked in each column by the same letters are not statistically different according to Duncan's multiple range test $(p=0.05, n=7)$.

Table 5. The influence of Bacillus velezensis BZR86 on the growth and development of cucumber plants (cv. Kurazh).

\begin{tabular}{|c|c|c|c|c|c|}
\hline \multirow{2}{*}{ Treatment } & \multirow{2}{*}{$\begin{array}{l}\text { Height } \\
\text { (cm) }\end{array}$} & \multirow{2}{*}{$\mathbf{N}^{\circ}$ Leaves } & \multicolumn{2}{|c|}{$\begin{array}{l}\text { Biomass Weight } \\
\text { (g) }\end{array}$} & \multirow{2}{*}{$\begin{array}{l}\text { Root Volume } \\
\quad(\mathrm{mL})\end{array}$} \\
\hline & & & Aerial Parts & Roots & \\
\hline $\begin{array}{l}\text { Non-infested } \\
\text { control }\end{array}$ & $51 \pm 10.9^{1} \mathrm{a}^{2}$ & $22 \pm 4.1 \mathrm{a}$ & $10.9 \pm 1.2 \mathrm{ab}$ & $4.2 \pm 1.5 \mathrm{a}$ & $7.6 \pm 2.2 \mathrm{~b}$ \\
\hline $\begin{array}{l}\text { Infested control } \\
\text { Phytoverm }\end{array}$ & $\begin{array}{c}48 \pm 10.4 \mathrm{a} \\
44 \pm 8.7 \mathrm{a}\end{array}$ & $\begin{array}{l}22 \pm 5.7 \mathrm{ab} \\
22 \pm 4.2 \mathrm{a}\end{array}$ & $\begin{array}{l}6.3 \pm 2.0 \mathrm{a} \\
6.1 \pm 3.8 \mathrm{a}\end{array}$ & $\begin{array}{l}4.1 \pm 2.1 \mathrm{a} \\
3.4 \pm 1.7 \mathrm{a}\end{array}$ & $\begin{array}{c}3.1 \pm 1.6 \mathrm{a} \\
6.0 \pm 2.4 \mathrm{ab}\end{array}$ \\
\hline $\begin{array}{l}\text { BZR } 86,7 \times 10^{6} \\
\text { CFU mL }{ }^{-1}\end{array}$ & $92 \pm 4.2 \mathrm{~b}$ & $27 \pm 1.8 \mathrm{~b}$ & $12.9 \pm 3.1 \mathrm{~b}$ & $6.6 \pm 2.1 \mathrm{a}$ & $6.0 \pm 2.8 \mathrm{ab}$ \\
\hline $\begin{array}{l}\text { BZR } 86,7 \times 10^{5} \\
\text { CFU mL }^{-1}\end{array}$ & $57 \pm 12.7 \mathrm{a}$ & $24 \pm 3.2 \mathrm{ab}$ & $9.6 \pm 2.6 \mathrm{ab}$ & $7.1 \pm 1.5 \mathrm{a}$ & $6.4 \pm 2.4 \mathrm{ab}$ \\
\hline $\begin{array}{l}\text { BZR } 86,7 \times 10^{4} \\
\text { CFU mL }^{-1}\end{array}$ & $50 \pm 13.7 \mathrm{a}$ & $22 \pm 3.4 \mathrm{ab}$ & $6.9 \pm 2.8 \mathrm{a}$ & $4.7 \pm 1.7 \mathrm{a}$ & $5.4 \pm 1.5 \mathrm{ab}$ \\
\hline
\end{tabular}

${ }^{1}$ Each value is an average \pm SD of seven replicates. ${ }^{2}$ Data flanked in each column by the same letters are not statistically different according to Duncan's multiple range test $(p=0.05, n=7)$.

In the experiment on tomato, application of $B$. velezensis BZR 86 almost completely eliminated the symptoms of RKND at all concentrations. Only single galls were formed on roots. At the lowest concentration of $B$. velezensis BZR $86\left(3 \times 10^{4} \mathrm{CFU} \mathrm{mL} \mathrm{m}^{-1}\right.$ of substrate), the gall numbers tended to increase, but the difference from other bacterial concentrations was not statistically significant (Table 4 ).

Application B. velezensis BZR 86 at $3 \times 10^{6}$ and $3 \times 10^{5} \mathrm{CFU} \mathrm{mL} \mathrm{m}^{-1}$ of substrate concentrations resulted in a significant increase in tomato root system biomass compared to chemical standard Phytoverm and non-infested soil, whereas this effect was absent at the lowest bacterium concentration. At the medium concentration of bacterium $\left(3 \times 10^{5} \mathrm{CFU} \mathrm{mL}^{-1}\right.$ soil), tomato root volume was significantly greater than at the lowest concentration and also than Phytoverm. It exceeded that parameter for the variant without RKN by four times (Table 4).

\section{Discussion}

The problem of root-knot disease on cucumber and tomato plants is really pressing in Russia, as most Russian farmers use soil substrates frequently infested by root-knot nematodes rather than hydroponics. At present, there are no microbial products registered against root-knot disease in the Russian Federation [44]. Chemical pesticide Phytoverm does not help to solve the problem when plant roots are strongly affected by the disease resulting in premature death of plants.

Bacillus species have been screened for nematicidal activity against RKNs [15,45,46], as well as for antifungal properties [47-49] and growth stimulation activity [50,51]. A number of studies documented a simultaneous activity of Bacillus strains against RKNs 
and fungi [19], as well as against RKNs or fungal phytopathogens, and its effect on plant growth enhancement $[11,16,52]$. In this study, we analyzed all these three parameters in the in vitro experiment, so as to select the most promising strain, i.e., B. velezensis BZR 86 isolated from winter wheat rhizosphere.

Previous research has shown a significant influence of genus Bacillus on mortality of juveniles of the root-knot nematode $M$. incognita [15,43-46,53,54]. Among others, $B$. velezensis had nematicidal activity against $M$. incognita [11,55-57]. In vitro $B$. velezensis BZR 86 caused $98 \%$ mortality of $M$. incognita $\mathrm{J} 2$ following a 24-h exposure to a $9 \times 10^{7} \mathrm{CFU} \mathrm{mL}^{-1}$ bacterial density [55]. In our study the nematicidal effect of B. velezensis BZR 86 was also confirmed on cucumber and tomato in soil, in agreement with previous studies that reported a significant suppressiveness of Bacillus strains on root-knot nematode eggs, J2 and root galls on cucumber [10,55], tomato [16,17,53,58], eggplant [15,59] and hendi [60]. The mechanisms underlying the RKN suppression by Bacillus strains can be different. Thus Burkett-Cadena et al. [17] suggested the production of antibiotic metabolites as responsible for soilborne pathogen suppression by B.subtilis GB03, while an induced systemic resistance (ISR) has been suggested as the main mechanism of biocontrol activity of B. amyloliguefaciens strain FZB42 [61].

Induced systemic resistance could also be a possible mechanism of $B$. velezensis BZR 86 suppressiveness on $M$. incognita, as some Bacillus strains were found to release jasmonic acid, known for reducing $M$. incognita infestation of tomato and cowpea by triggering plant defense against the root-knot nematode [61-64]. In good agreement, Toral et al. [63] reported significant increases in salicylic and jasmonic acid levels in strawberry plants treated with Bacillus velezensis XT1.

Bacillus strains were also found to produce nematicidal and antimicrobial compounds, including antibiotics, cyclic lipopeptides, polyketides and bacteriocins [23,65]. Since we observed a significant nematicidal effect of $B$. velezensis BZR 86 against M.incognita also in the in vitro assays, we may suppose that secondary metabolites of this strain could have a role in controlling RKND also in soil. Studies on genome of B. velezensis FZB 42 revealed that 13 gene clusters are responsible for the synthesis of predicted antimicrobial metabolites or volatile compounds [66].

Plant growth stimulation by $B$. velezensis BZR 86 observed in our experiments on cucumber and tomato is in good agreement with growth increase observed on olive trees treated with B. velezensis OEE1 [67]. Moreover, new isolated Bacillus strains were already stated for enhancing crop growth and productivity [65] and improving soil health [50,68,69]. Some bacilli are also known to fix $\mathrm{N}_{2}$ and soluble phosphate [70,71], thus promoting circulation of plant nutrients and, consequently, increasing crop growth and yield. Genes that contribute to plant growth promotion provide the possibility to use $B$. velezensis as a biofertilizer. It is known that bacilli produce plant growth promoting phytohormons (cytokinin, auxin) and volatile organic compounds (aceton (3-hydroxy-2-butanone), 2,3,butanediol) [52,72,73]. The significant increase in the root volume and root biomass of cucumber and tomato plants may be also due to locally reduced ethylene concentrations and increased assimilation of metal ions, such as iron, through the activation of plant's own iron acquisition mechanisms by members of the Bacillaceae [21]. Moreover, Qin et al. [68] documented a significant effect of B. amyloliguefaciens LS-60 on the structure of bacterial community associated with cucumber seedling. This change of community structure resulted in the dominance of genera Bacillus, Rhodanobacter, Paenibacillus, Pseudomonas, Nonomuraea and Agrobacterium, known for great impacts on soil nutritional composition, mineral metabolism and antibiotic production, thus providing an increased content of available nitrogen, phosphorus and potassium in soil substrate.

The number of bacteria in soil varies from $10^{7}$ to $10^{9}$ CFU per gram of soil [21]. We inoculated the bacterial formulations into soil substrate at concentrations far lower $\left(10^{4}-10^{6} \mathrm{CFU} \mathrm{mL}{ }^{-1}\right.$ of soil substrate) in order to minimize the effect on the ecosystem. However, future investigations are needed to analyze the mechanisms involved in the interaction among rhizospheric bacteria, plants and other components of soil ecosystem. There 
was no significant direct correlation between the concentration of bacterial formulations and development of RKND.

Our own results and data of other researchers $[29,47-49,64,67,74-77]$ demonstrated a strong antagonistic activity of $B$. velezensis to the pathogenic fungi Fusarium graminearum, F. oxysporum and R. solani, thus confirming this species as a very promising biocontrol agents $[24,78,79]$. Practical value of this antagonism has been proved by the use of $B$. velezensis in BioYield commercial formulation applied in management of soil-born pathogens and M. incognita on tomato [11].

The data obtained in this and further research will contribute to selection and comprehensive study of new biocontrol strains. They may form the basis for plant protection against plant parasitic nematodes as an alternative control measure. This also implies serious commercial potential, as the demand for such formulations is growing while global supply remains insufficient.

\section{Conclusions}

Selection of bacterial strains for control of RKND should be comprehensive and include analysis of nematicidal, fungicidal and growth stimulation activities. A number of bacterial strains with these characteristics are already present in the Bioresource Collection of FSCBPP that should be considered a valuable source of new innovative products for sustainable agricultural systems.

Bacterial strain B. velezensis BZR 86 showed to have a multiple effect on the complex of plants, RKNs and phytopathogenic microorganisms and, therefore, could be a potential candidate for the production of new biostimulants with a side suppressive activity on RKND. However, mechanisms of this multiple activity should be further investigated in detail, as to improve effects by optimizing techniques and timing of application.

Supplementary Materials: The following are available online at https://www.mdpi.com/article/ 10.3390/microorganisms9081698/s1, Table S1: Bacterial strains collected from Krasnodar region used in this study; Table S2: Enzymatic activity of bacterial strains from the Bioresource Collection of FSCBPP; Table S3: Effect of bacterial strains on growth and biomass of winter wheat plants in pot-experiment.

Author Contributions: Conceptualization, V.D.M. and A.M.A.; A.N.K. and S.V.L. performed experimental work with nematodes and plants; N.S.T. prepared figures and statistical analysis; V.M.D. conducted the experiments with microorganisms; writing—original draft preparation, V.D.M.; writing and editing, N.S. and T.D.; funding acquisition, A.M.A. All authors have read and agreed to the published version of the manuscript.

Funding: The study of bacterial strains was carried out within the framework of the State Assignment of the Ministry of Science and Higher Education of the Russian Federation, № 0686-2019-0013. DNA sequencing of the strain BZR 277, BZR 86 and the construction of the phylogenetic tree based on the whole genome sequencing was supported by a project of the Kuban Science Foundation (grant MFI-20.1/68).

Institutional Review Board Statement: Not applicable.

Informed Consent Statement: Not applicable.

Data Availability Statement: Data is contained within the article or Supplementary Material.

Acknowledgments: We thank Nikolay V. Ravin for helping with genome analysis. We express our gratitude to Anastasia Shchukovskaya for providing the culture of Paraphelenchus tritici.

Conflicts of Interest: The authors declare no conflict of interest.

\section{References}

1. Agrios, G.N. Plant diseases caused by nematodes. In Plant Pathology; Elsevier Academic Press: Burlington, NJ, USA, 2005; pp. 825-874.

2. Lychagina, S.V.; Shesteperov, A.A. Monitoring of root-knot disease of vegetable crops in greenhouses. Plant Prot. Quar. Plant. 2008, 4, 57-61. (In Russian) 
3. Johnson, A.W.; Littrell, R.H. Pathogenicity of Pythium aphanidermatum to Chrysanthemum in combined inoculations with Belonolaimus longicaudatus or Meloidogyne incognita. J. Nematol. 1970, 2, 255-259.

4. Mucksood, A.G.; Khan, T.A. Studies on the interactive effect of Meloidogyne incognita and Fusarium solani on Lycopersicon esculentum, Mill. Int. J. Bot. 2011, 7, 205-208. [CrossRef]

5. Lamelas, A.; Desgarennes, D.; López-Lima, D.; Villain, L.; Alonso-Sánchez, A.; Artacho, A.; Latorre, A.; Moya, A.; Carrión, G. The bacterial microbiome of Meloidogyne-Based Disease Complex in coffee and tomato. Front. Plant Sci. 2020, 11, 136. [CrossRef] [PubMed]

6. Singh, R.; Kumar, M.; Mittal, A.; Mehta, P.K. Microbial metabolites in nutrition, healthcare and agriculture. 3 Biotech. 2017, 7, 15. [CrossRef]

7. Zhou, D.; Feng, H.; Schuelke, T.; De Santiago, A.; Zhang, Q.; Zhang, J.; Luo, C.; Wei, L. Rhizosphere microbiomes from root knot nematode non-infested plants suppress nematode infection. Microb. Ecol. 2019, 78, 470-481. [CrossRef] [PubMed]

8. Glick, B.R. Introduction to Plant Growth-Promoting Bacteria. In Beneficial Plant-Bacterial Interactions; Springer International Publishing, Ed.; Springer: Heidelberg, Germany, 2020; pp. 1-37.

9. Munif, A.; Hallmann, J.; Sikora, R.A. The influence of endophytic bacteria on Meloidogyne incognita infection and tomato plant growth. J. ISSAAS 2013, 19, 68-74.

10. Kokalis-Burelle, N. Pasteuria penetrans for control of Meloidogyne incognita on tomato and cucumber, and M. arenaria on snapdragon. J. Nematol. 2015, 47, 207-213.

11. Xiang, N.; Lawrence, K.S.; Kloepper, J.W.; Donald, P.A.; McInroy, J.A.; Lawrence, G.W. Biological Control of Meloidogyne incognita by spore-forming plant growth-promoting rhizobacteria on cotton. Plant Dis. 2017, 101, 774-784. [CrossRef]

12. Mazzuchelli, R.C.L.; Mazzuchelli, E.H.L.; De Araujo, F.F. Efficiency of Bacillus subtilis for root-knot and lesion nematodes management in sugarcane. Biol. Control 2020, 143, 104185. [CrossRef]

13. Susič, N.; Žibrat, U.; Sinkovič, L.; Vončina, A.; Razinger, J.; Knapič, M.; Sedlar, A.; Širca, S.; Gerič Stare, B. From genome to field-observation of the multimodal nematicidal and plant growth-promoting effects of Bacillus firmus I-1582 on tomatoes using hyperspectral remote sensing. Plants 2020, 9, 592. [CrossRef]

14. Talavera, M.; Mizukubo, T.; Ito, K.; Aiba, S. Effect of spore inoculum and agricultural practices on the vertical distribution of the biocontrol plant-growth-promoting bacterium Pasteuria penetrans and growth of Meloidogyne incognita-infected tomato. Biol. Fertil. Soils 2002, 35, 435-440. [CrossRef]

15. Ashoub, A.H.; Amara, M.T. Biocontrol activity of some bacterial genera against root-knot nematode, Meloidogyne incognita. J. Am. Sci. 2010, 6, 321-328.

16. Zhao, D.; Zhao, H.; Zhao, D.; Zhu, X.; Wang, Y.; Duan, Y.; Xuan, Y.; Chen, L. Isolation and identification of bacteria from rhizosphere soil and their effect on plant growth promotion and root-knot nematode disease. Biol. Control 2018, 119, 12-19. [CrossRef]

17. Burkett-Cadena, M.; Kokalis-Burelle, N.; Lawrence, K.S.; van Santen, E.; Kloepper, J.W. Suppressiveness of root-knot nematodes mediated by rhizobacteria. Biol. Control 2008, 47, 55-59. [CrossRef]

18. Wolfgang, A.; Taffner, J.; Guimarães, R.A.; Coyne, D.; Berg, G. Novel strategies for soil-borne diseases: Exploiting the microbiome and volatile-based mechanisms toward controlling Meloidogyne-based disease complexes. Front. Microbiol. 2019, $10,1296$. [CrossRef]

19. Adam, M.; Heuer, H.; Hallmann, J. Bacterial antagonists of fungal pathogens also control root-knot nematodes by induced systemic resistance of tomato plants. PLoS ONE 2014, 9, e90402. [CrossRef]

20. Raymaekers, K.; Ponet, L.; Holtappels, D.; Berckmans, B.; Cammue, B.P.A. Screening for novel biocontrol agents applicable in plant disease management-A review. Biol. Control 2020, 144, 104240. [CrossRef]

21. Mandic-Mulec, I.; Stefanic, P.; van Elsas, J.D. Ecology of Bacillaceae. Microbiol. Spectr. 2015, 3, TBS-0017-2013. [CrossRef]

22. Kämpfer, P.; Busse, H.J.; Glaeser, S.P.; Kloepper, J.W.; Hu, C.H.; McInroy, J.A. Bacillus cucumis sp. nov. isolated from the rhizosphere of cucumber (Cucumis sativus). Int. J. Syst. Evol. Microbiol. 2016, 66, 1039-1044. [CrossRef]

23. Horak, I.; Engelbrecht, G.; van Rensburg, P.J.J.; Claassens, S. Microbial metabolomics: Essential definitions and the importance of cultivation conditions for utilizing Bacillus species as bionematicides. J. Appl. Microbiol. 2019, 127, 2. [CrossRef]

24. Rabbee, M.F.; Ali, M.S.; Choi, J.; Hwang, B.S.; Jeong, S.C.; Baek, K.H. Bacillus velezensis: A valuable member of bioactive molecules within plant microbiomes. Molecules 2019, 24, 1046. [CrossRef]

25. Migunova, V.D.; Sasanelli, N. Bacteria as biocontrol tool against phytoparasitic nematodes. Plants 2021, 10, 389. [CrossRef] [PubMed]

26. Ghahremani, Z.; Escudero, N.; Beltrán-Anadón, D.; Saus, E.; Cunquero, M.; Andilla, J.; Loza-Alvarez, P.; Gabaldón, T.; Sorribas, F.J. Bacillus firmus Strain I-1582, a nematode antagonist by itself and through the plant. Front. Plant Sci. 2020, 11, 796. [CrossRef]

27. Miljaković, D.; Marinković, J.; Balešević-Tubić, S. The significance of Bacillus spp. in disease suppression and growth promotion of field and vegetable crops. Microorganisms 2020, 8, 1037. [CrossRef]

28. Moscow State University. Methods of Soil Microbiology and Biochemistry; Zvyagintsev, D.G., Ed.; Moscow State University: Moscow, Russia, 1991; p. 304. (In Russian)

29. Asaturova, A.M.; Dubyaga, V.M.; Tomashevich, N.S. Selection of perspective biological control agents for fall wheat protection from fusarium diseases. Polythematic Online Sci. J. Kuban State Agrar. Univ. 2012, 75, 1-12. Available online: http:/ / ej.kubagro.ru/ 2012/01/pdf/37.pdf (accessed on 1 July 2021). 
30. KREWMNEVA, O.Y.; Asaturova, A.M.; Volkova, G.V. Selection of strains that are antagonistic to wheat leaf tan spot disease pathogen. Biotechnol. Russ. 2013, 5, 54-59.

31. Castaneda-Alvarez, C.; Aballay, E. Rhizobacteria with nematicide aptitude: Enzymes and compounds associated. World J. Microbiol. Biotechnol. 2016, 32, 203. [CrossRef]

32. Bergey, D.H.; Holt, J.G. Bergey's Manual of Systematic Bacteriology; Yi Hsien Publishing: Taiwan, China, 1984.

33. Sanger, F.; Nicklen, S.; Coulson, A.R. DNA sequencing with chain-terminating inhibitors. Proc. Natl. Acad. Sci. USA 1977, 84, 5463-5467. [CrossRef]

34. Asaturova, A.M.; Homyak, A.I.; Kozitsyn, A.E.; Shternshis, M.V.; Rakitin, A.L.; Beletsky, A.V.; Mardanov, A.V.; Ravin, N.V. Draft genome sequence of Bacillus velezensis BZR 277, a prospective biocontrol agent against phytoparasitic nematodes. Microbiol. Resour. Announc. 2021, 10, e00266-21. [CrossRef]

35. Radchenko, V.V.; Vasilyev, I.Y.; Ilnitskaya, E.V.; Garkovenko, A.V.; Asaturova, A.M.; Tomashevich, N.S.; Kozitsyn, A.E.; Milovanov, A.V.; Grigoreva, T.V.; Shternshis, M.V. Draft genome sequence of the plant growth promoting bacterium Bacillus subtilis strain BZR 517, isolated from winter wheat, now reclassified as Bacillus velezensis strain BZR 517. Microbiol. Resour Announc. 2020, 9 , e00853-20. [CrossRef]

36. Chaumeil, P.A.; Mussig, A.J.; Hugenholtz, P.; Parks, D.H. GTDB-Tk: A toolkit to classify genomes with the Genome Taxonomy Database. Bioinformatics 2020, 36, 1925-1927. [CrossRef] [PubMed]

37. Guindon, S.; Dufayard, J.F.; Lefort, V.; Anisimova, M.; Hordijk, W.; Gascuel, O.; Notes, A. New algorithms and methods to estimate maximum-likelihood phylogenies: Assessing the performance of PhyML 3.0. Syst. Biol. 2010, 59, 307-321. [CrossRef]

38. Asaturova, A.M.; Homyak, A.I.; Tomashevich, N.S.; Pavlova, M.D.; Zhevnova, N.A.; Dubyaga, V.M.; Kozitsin, A.Y.; Sidorova, T.M.; Nadykta, V.D.; Ismailov, V.Y. Conditions for the cultivation of new Bacillus bacteria being micro bioproduct producers. J. Pure Appl. Microbiol. 2015, 9, 2797-2804.

39. Montealegre, J.R.; Reyes, R.; Perez, L.M.; Herrera, R.; Silva, P.; Besoain, X. Selection of bioantagonistic bacteria to be used in biological control of Rhizoctonia solani in tomato. Electron. J. Biotechnol. 2003, 6, 116-127. [CrossRef]

40. Kado, C.I.; Heskett, M.G.; Langley, R.A. Studies on Agrobacterium tumefaciens: Characterization of strains 1D135 and B6, and analysis of the bacterial chromosome, transfer RNA and ribosomes for tumor inducing ability. Physiol. Plant Pathol. 1972, 2, 47-57. [CrossRef]

41. Shchukovskaya, A.G.; Shestepyorov, A.A.; Babosha, A.V.; Ryabchenko, A.S.; Tkachenko, O.B. Psychrotolerant mycohelminths Aphelenchus avenae, Aphelenchoides saprophilus, and Paraphelenchus tritici as potential bioagents against pink (Microdochium nivale) and speckled (Typhula ishikariensis) snow molds. In Proceedings of the International Conference Plant and Microbe Adaptations to Cold 2012, Sapporo, Japan, 24-28 June 2012; Hokkaido University: Sapporo, Japan, 2012; p. 87.

42. Shesteperov, A.A.; Savotikov, Y.F. Quarantine Phyto Helminthosis; Kolos: Moscow, Russia, 1995; pp. 180-181. (In Russian)

43. Püntener, W. Manual for Field Trials in Plant Protection, 2nd ed.; Agricultural Division, Ciba-Geigy Limited: Basel, Switzerland, 1981; p. 205.

44. Minselchos. State Catalogue of Pesticides and Agrochemicals Allowed for Use in the Russian Federation; Minselchos: Moscow, Russia, 2020; p. 148. (In Russian)

45. Chin Ann, Y. Screening for nematicidal activities of Bacillus species against root knot nematode (Meloidogyne incognita). J. Exp. Agric. Int. 2013, 3, 794-805.

46. Xia, Y.; Xie, S.; Ma, X.; Wu, H.; Wang, X.; Gao, X. The purL gene of Bacillus subtilis is associated with nematicidal activity. FEMS Microbiol. Lett. 2011, 322, 99-107. [CrossRef] [PubMed]

47. Zhu, Z.; Peng, Q.; Man, Y.; Li, Z.; Zhou, X.; Bai, L.; Peng, D. Analysis of the antifungal properties of Bacillus velezensis B-4 hrough a bioassay and complete-genome sequencing. Front. Genet. 2020, 11, 703. [CrossRef]

48. Wang, S.; Sun, L.; Zhang, W.; Chi, F.; Hao, X.; Bian, J.; Li, Y. Bacillus velezensis BM21, a potential and efficient biocontrol agent in control of corn stalk rot caused by Fusarium graminearum. Egypt. J. Biol. Pest Control 2020, 30, 9. [CrossRef]

49. Castro, D.; Torres, M.; Sampedro, I.; Martínez-Checa, F.; Torres, B.; Béjar, V. Biological control of Verticillium wilt on olive trees by the salt-tolerant strain Bacillus velezensis XT1. Microorganisms 2020, 8, 1080. [CrossRef]

50. Meng, Q.; Jiang, H.; Hao, J.J. Effects of Bacillus velezensis strain BAC03 in promoting plant growth. Biol. Control 2016, 98, 18-26. [CrossRef]

51. Balderas-Ruiz, K.A.; Bustos, P.; Santamaria, R.I.; González, V.; Cristiano-Fajardo, S.A.; Barrera-Ortiz, S.; Mezo-Villalobos, M.; Aranda-Ocampo, S.; Arturo Guevara-Garcia, A.A.; Galindo, E.; et al. Bacillus velezensis 83 a bacterial strain from mango phyllosphere, useful for biological control and plant growth promotion. AMB Expr. 2020, 10, 163. [CrossRef] [PubMed]

52. Moon, J.-H.; Won, S.-J.; Maung, C.E.H.; Choi, J.-H.; Choi, S.-I.; Ajuna, H.B.; Ahn, Y.S. Bacillus velezensis CE 100 inhibits root rot diseases (Phytophthora spp.) and promotes growth of japanese cypress (Chamaecyparis obtuse Endlicher) seedlings. Microorganisms 2021, 9, 821. [CrossRef]

53. Xiao, T.J.; Tan, S.Y.; Shen, Q.R.; Ran, W. Bacillus cereus X5 suppresses root-knot nematode of tomato by colonizing in roots and soil. Afr. J Microbiol. Res. 2012, 6, 2321-2327.

54. Zaghloul, R.A.; Neweigy, N.A.; Abou-Aly, H.E.; El-Sayed, S.A.; Bahloul, A.M. Nematicidal activity of some biocontrol agents against root-knot nematodes in-vitro. Res. J. Pharm. Biol. Chem. Sci. 2015, 6, 429-438. 
55. Migunova, V.D.; Konrat, A.N.; Lychagina, S.V.; Asaturova, A.M.; Sasanelli, N. Nematicidal effect of bacterial strain Bacillus sp. BZR 86 on larval mortality of the root-knot nematode. Contemporary parasitology-Major trends and challenges. In Proceedings of the International Conference VI Congress of the Society of Parasitologists, Saint Petersburg, Russia, 15-19 October 2018; p. 159.

56. Huang, W.K.; Cui, J.K.; Liu, S.M.; Kong, L.A.; Wua, Q.S.; Peng, H. Testing various biocontrol agents against the root-knot nematode (Meloidogyne incognita) in cucumber plants identifies a combination of Syncephalastrum racemosum and Paecilomyces lilacinus as being most effective. Biol. Control 2016, 92, 31-37. [CrossRef]

57. Choi, T.G.; Maung, C.E.H.; Lee, D.R.; Ajuna, H.B.; Lee, Y.S.; Kim, K.Y. Role of bacterial antagonists of fungal pathogens, Bacillus thuringiensis KYC and Bacillus velezensis CE 100 in control of root-knot nematode, Meloidogyne incognita and subsequent growth promotion of tomato. Biocontrol Sci. Technol. 2020, 30, 685-700. [CrossRef]

58. Colagiero, M.A.; Rosso, L.C.; Ciancio, A. Diversity and biocontrol potential of bacterial consortia associated to root-knot nematodes. Biol. Control 2018, 120, 11-16. [CrossRef]

59. El-Nagdi, W.M.A.; Abd-El-Khair, H. Application of Bacillus species for controlling root-knot nematode Meloidogyne incognita in eggplant. Bull. Natl. Res. Cent. 2019, 43, 154. [CrossRef]

60. Vetrivelkalai, P.; Sivakumar, M.; Jonathan, E.I. Biocontrol potential of endophytic bacteria on Meloidogyne incognita and its effect on plant growth in hendi. J. Biopestic. 2010, 3, 452-457.

61. Chowdhury, S.P.; Hartmann, A.; Gao, X.; Borriss, R. Biocontrol mechanism by root-associated Bacillus amyloliquefaciens FZB42-A review. Front. Microbiol. 2015, 6, 780. [CrossRef]

62. Pankaj, S.; Muttucumaru, N.; Powers, S.J.; Gaur, H.S.; Kurup, S.; Curtis, R.H.C. Differential defense response due to jasmonate seed treatment in cowpea and tomato against root-knot and potato cyst nematodes. Nematology 2013, 15, 15-21. [CrossRef]

63. Toral, L.; Rodríguez, M.; Béjar, V.; Sampedro, I. Crop protection against Botrytis cinerea by rhizhosphere biological control agent Bacillus velezensis XT1. Microorganisms 2020, 8, 992. [CrossRef]

64. Cao, Y.; Zhang, Z.; Ling, N.; Yuan, Y.; Zheng, X.; Shen, B.; Shen, Q. Bacillus subtilis SQR 9 can control Fusarium wilt in cucumber by colonizing plant roots. Biol. Fertil. Soil. 2011, 47, 495-506. [CrossRef]

65. Cao, Y.; Pi, H.; Chandrangsu, P.; Li, Y.; Wang, Y.; Zhou, H.; Xiong, H.; Helmann, J.D.; Cai, Y. Antagonism of two Plant-Growth Promoting Bacillus velezensis isolates against Ralstonia solanacearum and Fusarium oxysporum. Sci Rep. 2018, 8, 1-14. [CrossRef]

66. Pirttilä, A.M.; Mohammad Parast Tabas, H.; Baruah, N.; Koskimäki, J.J. Biofertilizers and biocontrol agents for agriculture: How to identify and develop new potent microbial strains and traits. Microorganisms 2021, 9, 817. [CrossRef] [PubMed]

67. Cheffi, M.; Bouket, A.C.; Alenezi, F.N.; Luptakova, L.; Belka, M.; Vallat, A.; Rateb, M.E.; Tounsi, S.; Triki, M.A.; Belbahri, L. Olea europaea L. Root endophyte Bacillus velezensis OEE1 counteracts oomycete and fungal harmful pathogens and harbours a large repertoire of secreted and volatile metabolites and beneficial functional genes. Microorganisms 2019, 7, 314. [CrossRef]

68. Qin, Y.; Shang, Q.; Zhang, Y.; Li, P.; Chai, Y. Bacillus amyloliquefaciens L-S60 reforms the rhizosphere bacterial community and improves growth conditions in cucumber plug seedling. Front. Microbiol. 2017, 8, 2620. [CrossRef]

69. Hashem, A.; Tabassum, B.; Abd-Allah, E.F. Bacillus subtilis: A plant-growth promoting rhizobacterium that also impacts biotic stress. Saudi J. Biol. Sci. 2019, 26, 1291-1297. [CrossRef]

70. Çakmakçi, R.; Donmez, F.; Aydın, A.; Şahin, F. Growth promotion of plants by plant growth-promoting rhizobacteria under greenhouse and two different field soil conditions. Soil Biol. Biochem. 2006, 38, 1482-1487. [CrossRef]

71. Bizos, G.; Papatheodorou, E.M.; Chatzistathis, T.; Ntalli, N.; Aschonitis, V.G.; Monokrousos, N. The role of microbial inoculants on plant protection, growth stimulation, and crop productivity of the olive tree (Olea europea L.). Plants 2020, 9, 743. [CrossRef] [PubMed]

72. Aeron, A.; Khare, E.; Jha, C.K.; Meena, V.S.; Aziz, S.M.A.; Islam, M.T.; Kim, K.; Meena, S.K.; Pattanayak, A.; Rajashekara, H.; et al. Revisiting the plant growth-promoting rhizobacteria: Lessons from the past and objectives for the future. Arch Microbiol. 2020, 202, 665-676. [CrossRef] [PubMed]

73. Zubair, M.; Hanif, A.; Farzand, A.; Sheikh, T.M.M.; Khan, A.R.; Suleman, M.; Ayaz, M.; Gao, X. Genetic screening and expression analysis of psychrophilic Bacillus spp. reveal their potential to alleviate cold stress and modulate phytohormones in wheat. Microorganisms 2019, 7, 337. [CrossRef]

74. Xu, W.; Zhang, L.; Goodwin, P.H.; Xia, M.; Zhang, J.; Wang, Q.; Liang, J.; Sun, R.; Wu, C.; Yang, L. Isolation, identification, and complete genome assembly of an endophytic Bacillus velezensis YB-130, potential biocontrol agent against Fusarium graminearum. Front. Microbiol. 2020, 11, 598285. [CrossRef]

75. Palazzinia, J.M.; Dunlapb, C.A.; Bowmanc, M.J.; Chulze, S.N. Bacillus velezensis RC 218 as a biocontrol agent to reduce Fusarium head blight and deoxynivalenol accumulation: Genome sequencing and secondary metabolite cluster profiles. Microbiol. Res. 2016, 192, 30-36. [CrossRef]

76. Grady, E.N.; MacDonald, J.; Ho, M.T.; Weselowski, B.; McDowell, T.; Solomon, O.; Renaud, J.; Yuan, Z.-C. Characterization and complete genome analysis of the surfactin-producing, plant-protecting bacterium Bacillus velezensis 9D-6. BMC Microbiol. 2019, 19, 5. [CrossRef]

77. Li, J.; Hu, M.; Xue, Y.; Chen, X.; Lu, G.; Zhang, L.; Zhou, J. Screening, identification and efficacy evaluation of antagonistic bacteria for biocontrol of soft rot disease caused by Dickeya zeae. Microorganisms 2020, 8, 697. [CrossRef] 
78. Mullins, A.J.; Li, Y.; Qin, L.; Hu, X.; Xie, L.; Gu, C.; Mahenthiralingam, E.; Xing Liao, X.; Webster, G. Reclassification of the biocontrol agents Bacillus subtilis BY-2 and Tu-100 as Bacillus velezensis and insights into the genomic and specialized metabolite diversity of the species. Microbiology 2020, 166, 1121-1128. [CrossRef]

79. Wang, C.; Zhao, D.; Qi, G.; Mao, Z.; Hu, X.; Du, B.; Liu, K.; Ding, Y. Effects of Bacillus velezensis FKM10 for promoting the growth of Malus hupehensis Rehd. and inhibiting Fusarium verticillioides. Front. Microbiol. 2020, 10, 2889. [CrossRef] 